\title{
PVS 98 Agent Models and their Application in Production Planning
}

\author{
Vladimir Mařík ${ }^{1}$, Michal Pěchouček ${ }^{1}$, Christophe Roche ${ }^{2}$ \\ marik@lab.felk.cvut.cz,pechouc@labe.felk.cvut.cz \\ Christophe.Roche@univ-savoie.fr
}

${ }^{1}$ The Gerstner Lab, CTU, FEE, Technická 2, CZ 16627 Prague 6, Czech Republic

${ }^{2}$ University of Savoie, LGIS Campus Scientifique, 73370 Le Bourget du Lac, France

\begin{abstract}
The paper describes a novel paradigm for production planning based on multiagent systems and its implementation. General models of the agents (so called PVS98 agents) developed for system integration purposes in the project-oriented manufacturing are presented in this paper. Whereas an agent architecture is described by means of KQML-like structures, a tri-base acquaintance model as a natural extension of the twin-base model is considered to formalize the negotiation and control processes. The particular instances of the models aimed at the production planning tasks in the TESLA-TV plant are shown.
\end{abstract}

\section{INTRODUCTION}

The growing complexity of problems to be solved in industry requires new overall software system architectures integrating the already existing "islands" of wellworking algorithms into more robust global systems. Co-operative multi-agent systems offer a novel approach to handle these complex integration tasks. A multiagent system (ideally) consists of a set of autonomous, mutually co-operating elements (agents) which communicate using a specific ACL (agent communication language). Typically, no central element is considered in a multi-agent community. Global behavior of the community is formed as a result of the individual agent's behavior and communication among them.

Integration architectures of multi-agent system usually involve a small number of well-developed agents with a clearly defined, highly specialized functionality. Each agent, as a rule, consists of a functional body (usually a stand-alone, already well running program with a precisely specified functionality) and a wrapper which is responsible for agent's engagement in the agents' community. The wrapper contains a model of the agent's behavior. The wrapper also "translates" the

The original version of this chapter was revised: The copyright line was incorrect. This has been corrected. The Erratum to this chapter is available at DOI: 10.1007/978-0-387-35390-6_58 
inter-agent ACL communication into the instructions for the activity of the functional body and mediates the results of the body activity into the agents' community. In order to ensure the most effective collaboration, agents are advised to maintain explicit models about their peer colleagues in order to select the bestqualified agent(s) for cooperation.

\section{EUREKA 1439 PROJECT AND TESLA-TV TESTBED}

The goal of the EUREKA No. 1439 - PVS 98 project $^{1}$ (started in 1996) is to propose a new approach for information handling and management of the projectoriented enterprises. This approach relies firstly on an agent modeling where each actor of an enterprise: person, department, factory, supplier, workshop, software module is viewed as an agent. Each of agents maintains skills and is able to communicate and collaborate with the others. Secondly, this approach is based on modeling knowledge, which is used, shared and exchanged among the community of agents. Research and development in the area of the agent-oriented and knowledge-oriented concurrent engineering and enterprise modeling and integration is motivated by number of either ongoing or finished projects: SHADE (SHAred Dependency Engineering) [McGuire 93], PACT (Palo Alto Collaborative Testbed) [Cutkosky 93], TOVE (Torento Virtual Enterprise) [Fox 92], Enterprise Project.

General models of the agents (so called PVS98 agents) and especially their wrappers developed for system integration purposes in the project-oriented manufacturing are presented in this paper. Whereas the communication architecture is described by means of KQML-like structures, a tri-base acquaintance model [Štěpánková, 97] as a natural extension of the twin-base model [Cao, 96] is considered to support the negotiation and control processes. The particular instances of the models aimed at the production planning tasks in the TESLA-TV plant are shown.

Each PVS98 agent is an autonomous entity, which allows encapsulating heterogeneous programs as agent's skills. An agent is defined by attributes and it communicates with the other agents using a KQML-like communication language. It contains its own negotiation and behavioral schemas represented in the form of production rules which are organized according to the tri-base acquaintance model. Communication and collaboration means transparently organized in the agents' wrappers, are the main features of the PVS98 agents.

\section{TRI-BASE ACQUAINTANCE MODEL}

A twin-base approach, a specific acquaintance model technique, was proposed recently [Cao 96]. This paradigm suggests organizing the relevant information

\footnotetext{
${ }^{1}$ EUREKA project participants: TESLA-TV company and Czech Technical University both in Prague, Czech Republic, AEGIS company and University of Savoy in France, EPFL in Lausanne, Giovanolla and Vevey Technolac in Switzerland.
} 
about co-operating agents in two separate information bases in the wrapper, namely in

- the COOPERATOR-BASE which has an auxiliary nature and which contains/collects information concerning the other agents. It can provide information on the data and message formats used by the other agents, their addresses, statistics concerning the other agents' efficiency, trust values etc.

- the TASK-BASE which contains relevant particular information on capabilities of the other agents in the form of the triples

$$
\left\langle T_{j}, A_{i}, S_{j i} \equiv\left\{\left\langle T_{j k}, A_{j k}\right\rangle\right\}\right\rangle
$$

where $T_{j}$ denotes the task, $A_{i}$ represents the name of the agent-coordinator and the set $S_{i j}$ of couples $\left\langle T_{j k}, A_{j k}\right\rangle$ expresses the sequence of sub-tasks to be solved under the coordination of the agent $A_{i}$. If the task $T_{j}$ can be solved by the agent $A_{i}$ itself, the set $S$ is empty.

The main innovative idea behind the twin-base approach is the opportunity to carry out periodical revisions of the contents of the task-bases by means of a special super-agent called a cooperation trader in [Cao 96]. Thus, the task-base always contains highly up-to-date information about the current capability of the others. This fact enables to direct the cooperation requests to the most suitable and applicable agents in the community. As a result, the communication traffic is significantly reduced and the reactions of the system are becoming substantially faster than if the broadcasting is used.

From the pure AI point-of-view, the triplets in a task-base can be interpreted as production rules containing knowledge. The cooperator-base contains important factual information and can be considered as a database.

The classical twin-base approach doesn't consider any complicated inference and solves the conflict resolution problem in a very simple way. The triplets are ordered according to the "trust" computable from history statistics.

In the tri-base approach, the original cooperator-base was split in two separate bases (the cooperator-base and the state-base) to explicitly distinguish between stable (permanent or semi-permanent) information in one hand (a cooperator-base) and the dynamically, very often up-dated and changed information on the community state on the other (a state-base).

Production system interpretation of the tri-base communication model calls for periodical specifying conditions under which the specific solution should be applied. To do so, it was proposed to use - instead of the triplets - the following quintuplets

$$
\left\langle T_{j}, A_{i} S_{j i} \equiv\left\{\left\langle T_{j k}, A_{j k}\right\rangle\right\}, C_{i j}, V_{i j}\right\rangle,
$$

where $C_{i j}$ represents a set of applicability constraints and $V_{i j}$ is a 'trust' of the agent $A_{i}$ in performing the task $T_{j}$ carrying out the sequence of sub-tasks $\left\{\left\langle T_{j k}, A_{j k}\right\rangle\right\}$.

From the production-system point of view, this quintuplet can be interpreted as a rule which is activated when the constraints $C_{i j}$ are satisfied. When a decision (choice among possible ways to solve the given task) is made in the wrapper of an agent (the corresponding rule is fired) using the task-base, a message is sent to the agent $A_{j 1}$ where $A_{j 1}$ is different from $A_{i}$. 
The message contains the triplet $\left\langle T_{j}, A_{i}, S_{i j}\right\rangle$ and the necessary additional information (namely relevant data) to start the solution process. When the sub-task $T_{j k}$ is solved by the agent $A_{j k}$, an analogous message is sent directly to the next agent $A_{j, k+1}$ in the set $S_{i j}$ to solve the sub-task $T_{j, k+1}$. When the sequence $S_{i j}$ cannot be solved completely (because some of the agents 'dies' in the meantime), the solution process is stopped and the task coordinating agent $A_{i}$ is informed and asked to make a new decision.

\section{STRUCTURES USED WITHIN PVS'98 AGENT}

There are four complementary structures to be considered when designing appropriate knowledge/data structures to represent agents' behavior:

a) wrapper internal structure,

b) interaction and coordination structure,

c) control structure.

\subsection{Internal Structure}

Agent's internal structure has to be represented within the wrapper frame. In principles, there are five obligatory slots involved in this substructure, namely

- pointer to the super-class in the agents' hierarchy (if any),

- list of actions the agent is capable to carry out,

- attributes and relations,

- list of local knowledge-bases, including the state-base of the tri-base acquaintance model,

- semantics of terminology used by the agent given by the corresponding ontology.

\subsection{Interaction Structure}

There are two aspects of agent's interaction - COMMUNICATION and COORDINATION. Message, a fundamental COMMUNICATION PRIMITIVE, is a frame-like structure consisting of three encapsulated layers described by a set of key words:

- The CONTENT LAYER contains the information transmitted between two agents: e.g. description of a product, of a task etc.

- The PERFORMATIVE LAYER specifies what the agent-sender expects from the agent-receiver. There is a number of various types of performatives: asking, replying, achieving and others. The notion of performative comes from the speech act theory describing general types of behaviour (ARPA Knowledge Sharing Effort projects [Patil 92]). We also introduce notion of priority, which is not included in KQML message structure.

- The COMMUNICATION LAYER provides all the information needed for performing communication transaction. The keyword TO indicates the agent receiver of the message which can either (1) be fully specified by its name, (2) described only by characteristic features (e.g. "every agent which owns such a skill' or 'agent belonging to a specific class'), or (3) unspecified - blind 
broadcasting. This introduces a "plug and play" approach to programming - a knowledge oriented type of programming.

Let's mention here, that the original architecture of the tri-base model considers existence of the task-coordinating agent that is responsible for supervising the solution of the given task till its completion. As the messages may contain a complete description of the sequence of tasks to be solved (the concept of continuation and delegation), the existence of such task coordinator is not necessary. The delegation message slot gives the receiver a piece of information who to delegate the task to, if the receiver fails to carry out the request. Such a case may arise from failing to understand the message, facing luck of resources, or handling an unpredicted situation. The continuation message slot contains information on which agent should continue in solving the problem. The COORDINATION structure contains information as follows:

- List of the input and output messages.

- Instructions how to treat the received messages. Such a treatment can be defined as a set of operations, which can be executed by local actions or in collaboration with the other agents. In the latter case, the treatment contains a negotiation schema - sending messages and waiting for replies has to be considered. This kind of knowledge is encoded within the task base of the agent tri-base model. Each treatment is expressed as a weighted production rule - IF conditions THEN actions with WEIGHT $p$, which corresponds exactly to the formalism of task base production quintuplets defined in [Štěpánkova 97].

- Knowledge/data bases supporting the interaction and coordination activities, e.g. the cooperator base, task base, resource base etc.

\subsection{Control Structure}

Two levels of the agent's control structure are responsible behavior of an agent. We distinguish between"

- MAILBOX LEVEL ADMINISTRATION, and

- MESSAGE LEVEL ADMINISTRATION.

The former level is in charge of handling received messages stored in the mailbox. When a new message arrives, the control algorithm must decide whether to process the incoming message immediately and thus suspend the current message processing with respect to the priority preference or whether to put the message in the waiting list. In the former case, new priority is assigned to each of suspended treatments and can be further on dynamically updated. By default, this priority is equal to the priority of the message, which originally fired the treatment.

The MESSAGE LEVEL ADMINISTRATION specifies the way, how to treat the currently processed message. Let us remark that in the special case of production planning the tri-base model is very often explored as a part of the agent's body (message content processing). The agent's tri-base acquaintance model is used to fire the appropriate treatment. The content of the message, applicability conditions based on the agent's status and status of collaborating agents and trust of the treatment must be considered. 


\section{TESLA-TV APPLICATION: PROPLANT SYSTEM}

TESLA TV is a Czech manufacturing enterprise producing TV transmitters, FM transmitters and passive elements. The manufacturing process in TESLA TV can be classified as a project oriented production. There is no conveyor-belt type of assembly line used and it is very difficult to formalize the production as a continuous process. The production goal is to manufacture (mainly assemble) a single particular transmitter. Consequently, rather than planning and simulation of a flow of semi-products to be assembled, there is a need for an information technology solution which would facilitate simple planning and subsequent optimization of the unique final product manufacturing.

Currently ill-suited information flows, lack of communication among particular production units and far from full utilization of available information processing solutions is what makes all of the production difficult to understand, model, plan and consequently optimize. Attempts to create a global, "monolithic" software solution, no matter how well hierarchically structured, have failed. There is a set of diverse software tools (from our point of view "legacy" software systems) used in the plant currently to solve same partial tasks. On one hand it is crucially important to integrate these partial solutions and on the other hand the flexibility and frequent changes in the production facility inevitably require highly distributed solutions. Multi-agent approach seems to be suitable in order to address this problem.

Analysis of information flows among departments of the Tesla TV enterprise strongly supported the choice of a multi-agent system as a suitable environment for building ProPlanT - Production Planning Tool. Its architecture is based on the following principles:

1) Any detailed project plan/schedule is prepared as a result of information exchange among finite number of agents (an agent is considered to correspond to factory information unit).

2) Each agent in the ProPlanT belongs to one of disjunctive classes of agents: PRODUCTION AGENTS - each production or store department is represented by this kind of "production" agent (PA) that models its behaviour/performance.

PRODUCTION MANAGERS - production management department is represented by autonomous production manager agents (PMAs).

PROJECT PLANNERS - a single Project Planning Agent (PPA) simulates functionality of the project, construction, and technology departments.

3) Agents are organized into a - in some sense - hierarchical structure (see Fig.1): The outputs of the production planner agent (PPA) appear first in the form of a set of tasks to be carried out and a set of precedence constraints for these tasks. Later, PPA structures the original set of tasks into a sequence of task groups in such a way that each group contains only those tasks which can be processed in parallel without violation of the fixed precedence constraints (for more details see [Maŕík 98]). The tasks from one group (those which can be fulfilled in parallel) are sent out to relevant PMAs according to the PMA 
specialization. PMA solves the task, if asked to, by further dividing it into jobs, which are mostly assigned to PAs.

4) A tri-base model can formalize the three used agent types, namely the PPA, PMA and PA. The cooperator-bases contain the permanent or semi-permanent knowledge on production facilities (their structure, transportation paths, capacity, etc.) as well as knowledge how to organize and plan projects. Part of the cooperator-base knowledge may be of heuristic nature. The state-bases contain temporary information on load, processing time, trust, etc. The taskbases are expected to store intermediate results of the planning process, i.e. the directives how to plan tasks and jobs.

5) Agents incorporated within the ProPlanT model communicate through KQML (knowledge query manipulation language) message with KIF (knowledge interchange format) content.

\section{PVS98 AGENT STRUCTURES IN PROPLANT}

The general PVS98 agent models can be represented by particular, more specific structures when applying the ProPlanT philosophy. The PVS98 agent modeling in ProPlanT relies on three agent classes: PPA, PMA and PA. In the agent ontology, all of them are subclasses of the super-class Agent.

In following we describe (1) message convention in terms of KQML performatives used and (2) each of the agent class used within the ProPlanT model. The explanation vehicle used in the latter case is an agent model structure described in Chapter 4.

\subsection{Messages}

There are three performatives used in the message passing process, namely

- ASK (an accusative performative) - a query to plan a possible production process,

- REQUEST (an imperative performative) - a request for triggering simulation of well specified production processes,

- REPLY/SORRY (an affirmative performative) - an answer reacting to one of above mentioned types of performatives. While REPLY reports on successful fulfillment of the requirement, SORRY reports on inability to do so.

The content of a message is either included into the message itself, or the content is to be transmitted through a shared database medium. That is why we distinguish between information-rich message, where the processed data are encapsulated within the message content, and information-free messages, where the only information within the message content is a pointer to the shared database and an appropriate filter.

\subsection{Production Agent (PA)}

On the PA level there is a local planning carried out through processing a distributed database of machines and other resources available to the given workshop. The jobs are scheduled if requested. 
A PA agent administers two taibles of a distributed, shared database medium - (1) its internal data specifying its responsibilities, times and costs of any operation it is capable to undertake and (2) a time database simulating the time-flow. The latter one gets updated when a request for simulation and scheduling is received. Talking in terms of the tri-base model, the former is encapsulated within the model's cooperator-base, whereas the latter one is stored within the state base of the model.

There are two kinds of messages the PA can accept: They are labeled by performatives ask and request in KQML. The message with the performative ask invokes the action query which offers a solution of the required task/job and estimates costs and deadlines. The message with the performative request triggers an execute action which does the same as the query, but simultaneously refines in the corresponding way the time database - updates the state base of the tri-base model. A PA agent can send a message with two kind of performative - reply and sorry, the function of which has been explained in Paragraph 6.1. The overall structure describing functionality of the PA agent is shown in the frame in Table 1:

\begin{tabular}{|c|c|c|c|c|c|}
\hline \multirow{3}{*}{$\sum_{\frac{2}{5}}^{\frac{\pi}{2}}$} & \multicolumn{2}{|r|}{ Praduction Agent (PA) } & \multicolumn{3}{|c|}{ SUPER-CLASS: AgEM } \\
\hline & \multicolumn{2}{|c|}{$\begin{array}{ll}\text { ACTION : } & \text { name : query } \\
& \text { parameters : (task or job) } \\
& \text { specification: duration and cost determination } \\
\text { ACTION : } & \text { name : execute } \\
& \begin{array}{l}\text { parameters : (task or job) } \\
\text { specification : adds the parameter to the agenda }\end{array}\end{array}$} & \multicolumn{3}{|c|}{$\begin{array}{l}\text { CHARACTERISTICS: } \\
\text { objectives : nil } \\
\text { roles : ( cost calculation, duration calculation, } \\
\quad \text { agenda update ) } \\
\text { state : (idle, working) }\end{array}$} \\
\hline & \multicolumn{3}{|c|}{ LOCAL KNOWLEDGE BASE: - state base: $\left[\left\langle\mathrm{M}_{\mathrm{i}}\right.\right.$, Capacity $_{\mathrm{i}}$, Trust $\left.\left._{\mathrm{i}}\right\rangle\right]$} & \multicolumn{2}{|l|}{ ONTOLOGY: nil } \\
\hline \multirow{4}{*}{$\frac{2}{3}$} & \multicolumn{2}{|c|}{ COLLABORATORS: $P A$} & \multicolumn{3}{|c|}{ COLLABORATE-TO:PA, PMA } \\
\hline & \multicolumn{2}{|c|}{$\begin{array}{l}\text { INPUT MESSAGES : } \\
\text { - performative : ask or request } \\
\text { - content : (task or job) } \\
\text { - sender : PA, PMA }\end{array}$} & \multicolumn{3}{|c|}{$\begin{array}{l}\text { OUTPUT MESSAGES: } \\
\text { - performative : reply or sorry } \\
\text { - content : (duration, cost) or a failure } \\
\text { - receiver: } P A, P M A\end{array}$} \\
\hline & \multicolumn{5}{|c|}{ 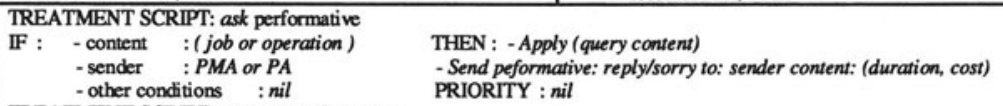 } \\
\hline & \multicolumn{4}{|c|}{$\begin{array}{l}\text { DELEGATION : - task base: }\left\{\left\langle\mathrm{O}_{\mathrm{j}}, \mathrm{PA}_{\mathrm{i}}, \mathrm{M}_{\mathrm{k}}, \mathrm{V}, \mathrm{C}\right\rangle\right\} \\
\quad-\text { cooperator base: }\left\{\left\langle\mathrm{O}_{\mathrm{j}}, \mathrm{M}_{\mathrm{k}}, \mathrm{V}, \mathrm{C}\right\rangle\right\} \& \text { rules for selection of optimal couple } \\
\langle\mathrm{O}, \mathrm{M}\rangle\end{array}$} & COMPLAINT : nil \\
\hline \multicolumn{6}{|c|}{$\begin{array}{l}\text { MAILBOX LEVEL ADMINISTRATION : FIFO - First in First out strategy } \\
\text { MESSAGE LEVEL ADMINISTRATION : tri-base acquaintance model }\end{array}$} \\
\hline
\end{tabular}

Behavior of a PA agent is given by a set of treatment instances based on general treatment scripts. During the preliminary planning the PA receives a requirement on sequence of jobs to be carried out, finds possible machines from the cooperator base, checks their current load and create acceptable quintuplets within the task base. At the operational planning, the currently most appropriate quintuplets in the task-base are chosen.

\subsection{Project Managing Agent (PMA)}

PMAs manage the process of project planning and simulation within the multiagent community. Each of PMAs is responsible (1) for a group of subordinated PAs and/or (2) a group of subordinated PMAs (if such a subordination has been 
created). Consequently the given PMA is supposed to be aware of agents it manages, about their abilities and limitation. At the same time it shall know the product structure in order to allocate tasks and delegate further responsibilities if necessary. There is a specific role a PMA plays within the multi-agent community. The operation of this agent is as follows:

- it uses its local knowledge in order to decompose the task into jobs (or task into tasks),

- it contracts its PAs (PMAs) with an appropriate request

Apart from the first data classification, that is not computationally expensive process, the manager is mainly engaged in contracting its workshops (PAs). The process of contracting the best possible peer is rooted in the tri-base model and it is identical to the case of PA, but the cooperator base monitors peer agents rather than machines. Functionality of the PVS98 PMA agent is shown in the frame depicted in Table 2:

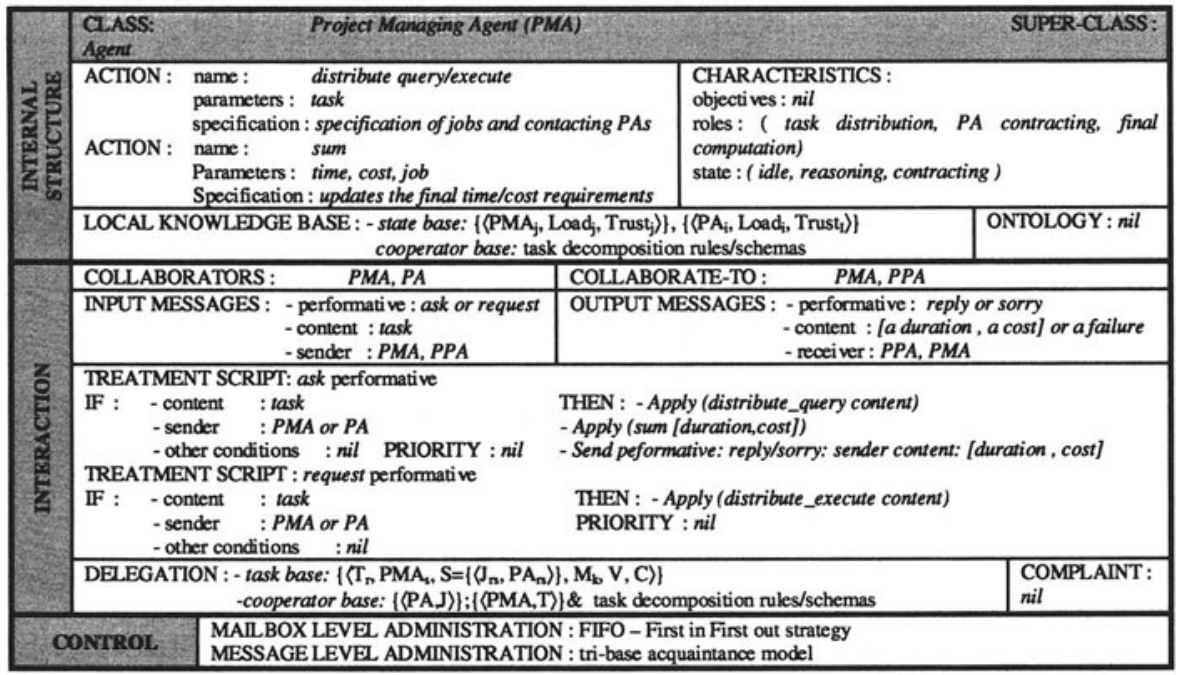

\subsection{Project Planning Agent (PPA)}

Project planning agent differs in structure and behavior from PA and PMA. In the case of project driven production it is quite tricky to specify thoroughly a product that would both satisfy needs of a customer and would be produced within certain cost limit and deadline. Certain performance/budget related compromise must be undertaken and considerable effort is usually devoted to translation of customer requirements into the 'language' of the enterprise.

The main tasks of the PPA agent is to analyze customer requirements, configure an acceptable solution in the form of a complete list of operations (jobs) to be carried out and a bill of material to be purchased. Problem solving knowledge of PPA are encoded within proof planning methods [Pěchouček 97]. 


\section{CONCLUSION}

There have been many papers published on multi-agent solutions recently. Typically, they are aimed either at theoretical/conceptual aspects of distributed artificial intelligence or at practical, ad hoc implementations of multi-agent systems. Our paper tries to contribute in filling the gap between both of these trends. First, generic structures and formalisms for a class of agents (PVS98 agents) are described and then a specific solution based on particular instances of the generic architectures is presented.

The entire system has been implemented in $\mathrm{C}++$ in Optima+t development environment. The system has been tested on parallel PC machines running Windows NT operating system. Bases of the agents' tri-base acquaintance model have been implemented in Sybase SQL Anywhere DBMS. And the PPA agent has been implemented in LPA Prolog

The research was carried out under the support of EUREKA No. 1439 project and the grant No. 102/96/0382 of the Czech Grant Agency (GAČR). We highly appreciate valuable comments of our colleagues Olga Štěpánková and Jiř́ Lažanský.

\section{REFERENCES}

[Cao 96] Cao W., Bian C.-G., Hartvigsen G.: Cooperator-Base and Task-Base for Agent Modeling: The Virtual Secretary Approach. In: Proc. of AAAI-96 Workshop on Agent Modeling, AAAI Press, 1996, pp. 105-111

[Cutkosky 93] Cutkosky M.R., Engelmore R.S., Fikes R., Gruber T.R., Genesereth M.R., Tenenbaum J.M., and Weber J.C., PACT: An experiment in integrating concurrent engineering systems, in IEEE Computer, vol. 26, No. 1, January 1993. [Fox 92] Fox M.S., The TOVE Project: Towards a Common Sense Model of the Enterprise, in Enterprise Integration, C. Petrie (Ed.), 1992, MA: MIT Press.

[McGuire 93], McGuire R., Kuokka D., Weber J.C., Tenenbaum J.M., Gruber T.R. Olsen R.G., SHADE:Technology for Knowledge-Based Collaborative Engineering, Concurrent Engineering: Research \& Applications, vol. 1, No. 3, September 1993

[Mařík 98] Mařík V., Pěchouček M., Hazdra T., Štěpánková O.: ProPlanT-MultiAgent System for Production Planning. in Proc. of the XIVth European Meeting on Cybernetics and Systems Research, Vienna, 1997, pp. 725-730

[Patil 92] Patil R., et al. The DARPA Knowledge Sharing Effort: Progress report. In: Principles of Knowledge Representation and Reasoning: Proc. of the Third International Conference, Rich C., et al, Ed., Cam., MA, Morgan Kaufmann, 1992. [Pěchouček 97] Pěchouček M., Lowe H., Bundy A.: Proof Planning and Industrial Configuration, Proceedings of the Fifth Conference of Practical Application of Prolog, PAC, London, April pp. 233 - 241, 1997.

[Štěpánková 97] Štěpánková O., Mařík V., Lažanský J.: Role of Qualitative Reasoning in a Multi-agent System. In Computer Aided System Theory, Pichler, F. Moreno-Diaz, R. eds., LNCS No.:1339, Springer-Verlag, Heidelberg, 1997

[Wooldridge 95] Wooldridge M., Jennings N.: Intelligent Agents: Theory and Practice. The Knowledge Engineering Review, 10 (1995), No.2, pp. 115-15 